\title{
RECONSTRUCTION OF FREEFORM SURFACE BY SUPPORT VECTOR REGRESSION
}

\author{
Ling Jing and Ling Zhen \\ Science of College, China Agriculture University, Beijing, 100083,China
}

\begin{abstract}
Reconstruction and manufacturing of existing freeform surfaces are of paramount importance for reverse engineering. The paper presents support vector regression (SVR) to reconstruction of computer models for existing freeform surfaces. Through examples, the effective is compared among different methods, and the influence of kernels to the precision is discussed. The results show that SVR is better than the algorithms given in [3] and [4], when it is used to reconstruct freeform surface.
\end{abstract}

Key words: reverse engineering, freeform surface, support vector regression, kernel

\section{INTRODUCTION}

One of the main issues in reverse engineering is to generate models or design representations based on the existing products ${ }^{[1-2]}$. There are a variety of engineering applications. For example, in the automobile, shipbuilding, or aircraft industries, designers may create a physical model based on the functional requirements and analysis. The model does not have a mathematical representation. For simple geometry, it is not difficult to generate such a representation in terms of drawings although it is very time consuming. If the model has very complex surface such as freeform surface, the development of such design representations or models becomes difficult.

A neural network approach is presented in [3]. To evaluate the effectiveness of the approach, a known non-uniform B-spline surface was 
used for generating a number of samples for training the networks. There 4layered neural networks were designed and trained using a modified back propagation algorithm. Taking advantage of the global minimum property of Simulated Annealing Procedure, a new technique ${ }^{[4]}$ is proposed to accept a temporally failed training result in accordance to probability. This method is able to jump out of the local minimum and converge to the global minimum in some cases, but easily leads to the problem of over-fitting .

Support vector machines have become a subject of intensive study. They have been applied successfully to classification and also to regression. In this paper we use support vector machines in the field of the reconstruction of freeform surfaces and we find that they show an excellent performance.

In the following sections we will introduce the basic principles of $\varepsilon$ support vector regression ( $\varepsilon$-SVR) and $v$-support vector regression $(v-$ SVR) in reconstruction of freeform surfaces. The experimental section considers a comparison of $\varepsilon$-SVR, $v$-SVR and other methods. Furthermore we compare the influence of different types of kernel function. A brief discussion concludes the paper.

\section{2. $\varepsilon$-SVR AND $v$-SVR}

In order to model and machine an existing freeform surface, the freeform surface is first digitized by sampling a number of points. Our task is to find a regression function based on the digitized sample points - the training points.

Suppose the training data are given by a training set $\mathrm{S}$ of labeled inputs

$$
S=\left\{\left(U_{1}, P_{1}\right),\left(U_{2}, P_{2}\right), \cdots,\left(U_{l}, P_{l}\right)\right\}
$$

For $i=1, \cdots, l$, the input $U_{i}=\left(u_{i}, v_{i}\right) \in R^{2}$ is the parameter of digitized point $P_{i}=\left(x_{i}, y_{i}, z_{i}\right) \in R^{3}$ of the freeform surface.

A parametric surface $p(u, v)$ can be decomposed into three surfaces $x(u, v), y(u, v)$ and $z(u, v)$ if the surface is a function of the parametric variables $u$ and $v$. To reconstruct the surface in terms of points, we are interested in the relationships between the parametric variables $u, v$ and the coordinate values of $x, y$ and $z$. Thus, SVR process is designed as three separate process corresponding to the three functions $x(u, v), y(u, v)$ and $z(u, v)$. We will only describe the process to find $x(u, v)$ below.

The input $U=(u, v)$ is first mapped to a feature space by a function $\Phi$. Assume that regression function $x(u, v)$ takes the following form:

$$
f(U)=(w \cdot \Phi(U))+b
$$

where $\Phi: R^{2} \rightarrow H, H$ is a Hilbert space, $w \in H, b \in R$. Let the loss function is the $\varepsilon$-insensitive loss function 


$$
c(U, x, f(U))=\widetilde{c}(f(U)-x)=|x-f(U)|_{\varepsilon}=\max \{0,|x-f(U)|-\varepsilon\}
$$

Where $\varepsilon>0$. This function ignores errors that are within a certain distance of the true value.

Suppose that $\varepsilon$ is given in priori, the primal problem is $\left.{ }^{[5-6}\right]$ :

$$
\begin{aligned}
& \min _{w, b, \xi, \xi^{*}} \tau\left(w, b, \xi_{i}, \xi_{i}^{*}\right)=\frac{1}{2}\|w\|^{2}+C \cdot \frac{1}{l} \sum_{i=1}^{l}\left(\xi_{i}+\xi_{i}^{*}\right) \\
& \text { s.t. }\left\{\begin{array}{l}
\left(w \cdot \Phi\left(U_{i}\right)\right)+b-x_{i} \leq \varepsilon+\xi_{i} \\
x_{i}-\left(w \cdot \Phi\left(U_{i}\right)\right)-b \leq \varepsilon+\xi_{i}^{*} \\
\xi_{i}, \xi_{i}^{*} \quad \geq 0
\end{array} \quad(i=1, \ldots, l)\right.
\end{aligned}
$$

where $\xi=\left(\xi_{1}, \xi_{2}, \cdots, \xi_{l}\right)^{\mathrm{T}}, \xi^{*}=\left(\xi_{1}^{*}, \xi_{2}{ }^{*}, \cdots, \xi_{l}{ }^{*}\right)^{\mathrm{T}} . C \geqslant 0, \varepsilon>0$ are constants . In order to solve problem (4), SVR introduces its dual problem

$$
\begin{array}{ll}
\min _{\alpha_{i}, \alpha_{i}^{*}} \quad \frac{1}{2} \sum_{i, j=1}^{l}\left(\alpha_{i}^{*}-\alpha_{i}\right)\left(\alpha_{j}^{*}-\alpha_{j}\right) K\left(U_{t}, U_{j}\right)+\varepsilon \sum_{i=1}^{l}\left(\alpha_{i}^{*}+\alpha_{i}\right)-\sum_{i=1}^{l}\left(\alpha_{i}-\alpha_{i}^{*}\right) x_{i} \\
\text { s.t. }\left\{\begin{array}{l}
\sum_{i=l}^{l}\left(\alpha_{i}-\alpha_{i}^{*}\right)=0 \\
\alpha_{i}, \alpha_{i}^{*} \in[0, C / l], \quad(i=1, \ldots, l)
\end{array}\right.
\end{array}
$$

Where $\alpha=\left(\alpha_{1}, \alpha_{2}, \cdots, \alpha_{i}\right)^{\mathrm{T}}, \alpha^{*}=\left(\alpha_{1}{ }^{*}, \alpha_{2}{ }^{*}, \cdots, \alpha_{1}{ }^{*}\right)^{\mathrm{T}}, K\left(U_{i}, U_{j}\right)=\left(\Phi\left(U_{i}\right)\right.$ ,$\left.\Phi\left(U_{j}\right)\right)$ is the kernel function. After getting the solution $\alpha$ and $\alpha^{*}$ of problem (5), we obtain the decision function as

$$
f(U)=\sum_{i=1}^{l}\left(\alpha_{i}^{*}-\alpha_{i}\right) K\left(U_{i}, U\right)+b=\sum_{S V}\left(\alpha_{i}^{*}-\alpha_{i}\right) K\left(U_{i}, U\right)+b
$$

Where $b$ is determined by KKT conditions. SV means the support vectors.

It must be pointed out that if $U_{i}$ satisfies to $\left|f\left(U_{i}\right)-x_{i}\right|<\varepsilon, \quad \alpha_{i}, \alpha_{i}^{*}$ must be zero by the KKT conditions. This means $w$ in (2) relies only on the $U_{i}$ with $\alpha_{i}-\alpha_{i}^{*} \neq 0$. We call these points the support vectors, this property makes SVR be applied in pre-handle the data in reverse engineering.

Although the parameter $\varepsilon$ does control the sparseness of the solution, it does this only in a rather indirect way. Lacking a priori information about the accuracy of the $x$-values, it can be difficult to come up with a reasonable value of $\varepsilon$ a priori. Instead, one would rather specify the degree of sparseness and let the algorithm compute $\varepsilon$ from the data. This is the idea of the $v-\mathrm{SVM}^{[5-6]}$, a modification of the original $\varepsilon$-SVM. In the $v$-SVM the size of $\varepsilon$ is not defined a priori but is itself a variable. Its value is traded off against model complexity and slack variables via a constant $v$ : 


$$
\begin{aligned}
& \min _{w, b, \xi, \xi^{*}, \varepsilon} \quad \begin{aligned}
\tau\left(w, b, \xi_{i}, \xi_{i}^{*}, \varepsilon\right) & =\frac{1}{2}\|w\|^{2}+C\left(v \varepsilon+\frac{1}{l} \sum_{i=1}^{1}\left(\xi_{i}+\xi_{i}^{*}\right)\right)
\end{aligned} \\
& \text { s.t. }\left\{\begin{aligned}
\left(w \cdot U_{i}\right)+b-x_{i} & \leq \varepsilon+\xi_{i} \\
x_{i}-\left(w \cdot U_{i}\right)-b_{i} & \leq \varepsilon+\xi_{i}^{*} \\
\xi_{i}, \xi_{i}^{*} \geq 0, \quad \varepsilon & \geq 0
\end{aligned}\right.
\end{aligned}
$$

where $C>0,0 \leq v \leq 1$ are constants. The dual problem of problem (7) is

$$
\begin{array}{ll}
\min _{\alpha_{i}, \alpha_{i}^{*}} \quad \frac{1}{2} \sum_{i, j=1}^{l}\left(\alpha_{i}^{*}-\alpha_{i}\right)\left(\alpha_{j}^{*}-\alpha_{j}\right) K\left(U_{i}, U_{j}\right)-\sum_{i=1}^{l}\left(\alpha_{i}^{*}-\alpha_{i}\right) x_{i} \\
\text { s.t. } \quad\left\{\begin{array}{l}
\sum_{i=1}^{l}\left(\alpha_{i}-\alpha_{i}^{*}\right)=0 \\
\alpha_{i}, \alpha_{i}^{*} \in[0, C / l] \\
i \\
\sum_{i=1}^{l}\left(\alpha_{i}+\alpha_{i}^{*}\right) \leq C \cdot v
\end{array}\right.
\end{array}
$$

We will use the term errors to refer to training points lying outside of the $\varepsilon$-tube, and the term fraction of errors/SVs to denote the relative numbers of errors/SVs, i.e. divided by $l$. This has been proved that $v$ can be used to control the fraction of support vectors (and hence the sparsity of the solution's expansion) and the fraction of outliers (i.e. the amount of confidence that we have in the data).

\section{RECONSTRUCTION OF SURFACE}

The example in [3-4] is now used to evaluate the effectiveness of SVR. Applying $\varepsilon-S V R$ and $v-S V R$, the reconstruction of the existing surface is realized by first training a series of points from a mathematically known surface in [3], the results were severed as models to generate all the points that are needed. The input is the two parametric variables $U=(u, v)$ of sample point. The output is the point of reconstruction surface. The expected output is the accurate point calculated using the NURBS equations. Then, we calculated a number of testing points using other set of $U=(u, v)$, the points and the differences between the points output from the predicting and the points generated by the NURBS equations indicated the performance of the different ways.

In learning process, the kernel form is chosen as: 


$$
K\left(U_{i}, U_{j}\right)=\left(\left(U_{i} \cdot U_{j}\right)+1\right)^{5}
$$

For the sake of simplicity, only the $x$-coordinate result is generated by different methods in Table 1. There $x_{1}$ is accurate value in the known surface, $x_{2}$ and $x_{3}$ are the results in [3] and [4], $x_{4}$ is given by $\varepsilon-S V R$ with the capacity constant $C=225$ and $\varepsilon=0.01$, and $x_{5}$ is obtained by $v-S V R$ with the capacity constant $C=225$ and $v=0.9$.

Table 1. $x$-coordinate generated by different methods

\begin{tabular}{|c|c|c|c|c|c|c|}
\hline$u$ & $v$ & $x_{1}$ & $x_{2}$ & $x_{3}$ & $x_{4}$ & $x_{5}$ \\
\hline 1.000000 & 1.000000 & 11.500000 & 11.447000 & 11.487403 & 11.5035 & 11.5099 \\
\hline 1.000000 & 1.100000 & 12.264167 & 12.275300 & 12.266978 & 12.2589 & 12.2529 \\
\hline 1.000000 & 1.200000 & 13.053333 & 13.102600 & 13.042305 & 13.0473 & 13.0398 \\
\hline 1.000000 & 1.300000 & 13.862500 & 13.928400 & 13.870028 & 13.8597 & 13.8562 \\
\hline 1.000000 & 1.400000 & 14.686677 & 14.752000 & 14.706395 & 14.688 & 14.6902 \\
\hline 1.000000 & 1.500000 & 15.520833 & 15.572800 & 15.547921 & 15.5252 & 15.5322 \\
\hline 1.000000 & 1.600000 & 16.360000 & 16.390100 & 16.391150 & 16.365 & 16.3744 \\
\hline 1.000000 & 1.700000 & 17.199167 & 17.203300 & 17.232766 & 17.202 & 17.2113 \\
\hline 1.000000 & 1.800000 & 18.033333 & 18.011800 & 18.048636 & 18.0321 & 18.0386 \\
\hline 1.000000 & 1.900000 & 18.857500 & 18.815000 & 18.875552 & 18.8518 & 18.8539 \\
\hline 1.000000 & 2.000000 & 19.666667 & 19.612400 & 19.717749 & 19.6586 & 19.6558 \\
\hline 1.000000 & 2.100000 & 20.457167 & 20.403300 & 20.427167 & 20.4511 & 20.444 \\
\hline 1.000000 & 2.200000 & 21.230667 & 21.187200 & 21.215274 & 21.2287 & 21.2192 \\
\hline 1.000000 & 2.300000 & 21.990167 & 21.963600 & 21.989935 & 21.9918 & 21.9826 \\
\hline 1.000000 & 2.400000 & 22.738677 & 22.732100 & 22.736344 & 22.7418 & 22.736 \\
\hline 1.000000 & 2.500000 & 23.479167 & 23.492200 & 23.483185 & 23.481 & 23.4815 \\
\hline 1.000000 & 2.600000 & 24.214667 & 24.243400 & 24.299485 & 24.2126 & 24.2212 \\
\hline 1.000000 & 2.700000 & 24.948167 & 24.985300 & 24.964167 & 24.9409 & 24.9571 \\
\hline 1.000000 & 2.800000 & 25.682667 & 25.717500 & 25.667085 & 25.671 & 25.6911 \\
\hline 1.000000 & 2.900000 & 26.421167 & 26.439700 & 26.417486 & 26.4092 & 26.4242 \\
\hline 1.000000 & 3.000000 & 27.166667 & 27.151500 & 26.945260 & 27.1625 & 27.1571 \\
\hline 1.100000 & 1.000000 & 11.500000 & 11.446900 & 11.430027 & 11.5029 & 11.5105 \\
\hline 1.100000 & 1.100000 & 12.264167 & 12.275200 & 12.269364 & 12.2588 & 12.2529 \\
\hline 1.100000 & 1.200000 & 13.053333 & 13.102600 & 13.044518 & 13.0475 & 13.0394 \\
\hline 1.100000 & 1.300000 & 13.862500 & 13.928400 & 13.871958 & 13.8603 & 13.8555 \\
\hline 1.100000 & 1.400000 & 14.686677 & 14.752000 & 14.708116 & 14.6889 & 14.6893 \\
\hline 1.100000 & 1.500000 & 15.520833 & 15.572800 & 15.549450 & 15.5262 & 15.5312 \\
\hline 1.100000 & 1.600000 & 16.360000 & 16.390200 & 16.392507 & 16.3661 & 16.3735 \\
\hline 1.100000 & 1.700000 & 17.199167 & 17.203400 & 17.233973 & 17.2032 & 17.2104 \\
\hline 1.100000 & 1.800000 & 18.033333 & 18.011900 & 18.030717 & 18.0334 & 18.0379 \\
\hline 1.100000 & 1.900000 & 18.857500 & 18.815200 & 18.899829 & 18.8531 & 18.8534 \\
\hline 1.100000 & 2.000000 & 19.666667 & 19.612500 & 19.718640 & 19.66 & 19.6555 \\
\hline 1.100000 & 2.100000 & 20.457167 & 20.403500 & 20.424773 & 20.4526 & 20.444 \\
\hline 1.100000 & 2.200000 & 21.230667 & 21.187400 & 21.216083 & 21.2303 & 21.2194 \\
\hline 1.100000 & 2.300000 & 21.990167 & 21.963900 & 21.990731 & 21.9935 & 21.983 \\
\hline 1.100000 & 2.400000 & 22.738677 & 22.732400 & 22.747147 & 22.7436 & 22.7367 \\
\hline 1.100000 & 2.500000 & 23.479167 & 23.492500 & 23.514025 & 23.4829 & 23.4824 \\
\hline 1.100000 & 2.600000 & 24.214667 & 24.243700 & 24.300312 & 24.2147 & 24.2224 \\
\hline
\end{tabular}




\begin{tabular}{lllllll}
1.100000 & 2.700000 & 24.948167 & 24.985600 & 24.995192 & 24.9432 & 24.9585 \\
1.100000 & 2.800000 & 25.682667 & 25.717900 & 25.668068 & 25.6736 & 25.6926 \\
1.100000 & 2.900000 & 26.421167 & 26.440100 & 26.418542 & 26.412 & 26.4259 \\
1.100000 & 3.000000 & 27.166667 & 27.152000 & 26.946396 & 27.1655 & 27.1589 \\
max-error & & & 0.0659 & 0.221407 & 0.011967 & 0.0144 \\
min-error & & 0.004133 & 0.000232 & $3.3 \mathrm{e}-5$ & 0.001967 \\
average-error & & 0.034621 & 0.033148 & 0.004391 & 0.008415 \\
\hline
\end{tabular}

The last three rows in Table $l$ indicate the maximum error, minimum error and the average error between the predicted value and the true value. The results show that the SVR approach is more effective than the methods used in [3] and [4].

\section{DISCUSSION THE KERNEL}

The use of the kernel function is the key of SVR to the reconstruction of the freeform surface. In order to observe the influence of different forms of kernel to approximate surface, different kernels are used, and the related results are showed in Table 2.Here we use $\varepsilon-S V R$ with the capacity constant $C=225$ and $\varepsilon=0.01$.

Table 2. the influence of different kernels

\begin{tabular}{lllll}
\hline kernel function & & max-error & min-error & average-error \\
\hline dot kernel & & 0.371167 & 0.0005 & 0.179011 \\
\hline \multirow{2}{*}{ polynomial kernel } & $\mathrm{d}=2$ & 0.1468 & 0.002 & 0.054185 \\
& $\mathrm{~d}=5$ & 0.018367 & 0 & 0.007824 \\
\hline \multirow{2}{*}{ radial kernel } & $\gamma=2$ & 0.792433 & 0.0033 & 0.185621 \\
& $\gamma=4$ & 0.634733 & 0.001267 & 0.142206 \\
\hline \multirow{2}{*}{ hyperbolic } & $a=1, b=1$ & 251.4227 & 2.1898 & 94.74889 \\
kernel & $a=0.0001, b=1$ & 0.294233 & 0.008967 & 0.110213 \\
& $a=0.0001, b=0.0001$ & 0.283133 & 0.012667 & 0.107197 \\
\hline
\end{tabular}

\section{CONCLUSION}

One of challenges in reverse engineering is to reconstruct computer representations of existing surfaces, based on digitized points. The problem becomes even more difficult when a surface is partially damaged or worn. Surface fitting is an obvious technique, however, to fit an existing surface, appropriate surface equations are first selected, and then the equations are evaluated to determine whether the approximation is acceptable. Selecting and evaluating the models remain difficult tasks. It will be difficult to use the surface fitting technique if the surface is partially damaged or worn or incompleted. 
In this work we show that SVR can be used to reconstruction the freeform surfaces in reverse engineering. It not only gives the formula of the freeform surface but also predicts the position of input parameters which aren't be solved in [3-4].

The parameters $\varepsilon$ and $v$ provide the user to construct freeform surface and control the errors at the same time. How to given the appropriate forms of kernel function is worth studying for the future.

\section{REFERENCES}

[1] Bolle R M, Vemuri B C. On three-dimensional surface reconstruction methods. IEEE Transactions on Pattern Analysis and Machine Intelligence, 1991, 13 (1): 1 13

[2] Varady T, Martin R R, and Cox J. Reverse engineering of geometric models-an introduction. Computer Aided Design, 1997, 29 (4): 255 268

[3] Gu P, Yan X. Neural network approach to the reconstruction of freeform surface for reverse engineering. Computer-Aided Design, 1995, 27: 59 64

[4] Wang K, Zhang C M, Neural network method to reconstruct the freeform surface. Journal of Computer-Aided Design \& Computer Graphics, 1998, 10 (3): 193 199

[5] Nello Cristianini and John Shawe-Taylor. An introduction to Support vector machines. Cambridge University Press, Cambridge,UK,2000

[6] Deng N Y, Tian Y J. The new method in data mining- Support Vector Machine. Science Press, Beijing, China, 2004 